九州大学学術情報リポジトリ

Kyushu University Institutional Repository

\title{
Random frequency process
}

Onoyama, Takuj i

https://doi.org/10.5109/12956

出版情報: 統計数理研究. 5 (1/2)，pp.51-58，1952-09. Research Association of Statistical Sciences

バージョン：

権利関係 : 


\title{
RANDOM FREQUENCY PROCESS
}

\author{
Takuji ONOYAMA
}

\section{Introduction.}

Let $(\boldsymbol{Q}, \mathfrak{F}, P$ ) be a probability field, $\Omega, \mathfrak{F}$, and $\boldsymbol{P}$ being an abstract set, a completely additive class of subsets of $Q$, and a Lebesque measure defined on $\mathfrak{F}$ respectively.

In harmonic analysis of a stochastic process, the following special class of a stationary process may be obtained:

$$
x(t, \omega)=\sum_{n=1}^{\infty} a_{n t}(\omega) e^{i \lambda, t}
$$

where

i) $\left\{a_{n}(\omega)\right\}(n=1,2, \cdots \cdots)$ is a sequence of mutually independent real random variables defined on $(\Omega, \mathfrak{F}, P)$;

ii) $\left\{\lambda_{n}\right\}(n=1,2, \cdots \cdots)$ is some sequence of real numbers, and $t$ is a continuous time parameter;

iii) $E\left\{\boldsymbol{a}_{n}(\omega)\right\}$ denotes the mathematical expectation of a random variable $a_{n}(\omega)$ and we suppose that $\sum_{n=1}^{\infty} E\left\{a_{n}{ }^{2}(\omega)\right\}<\infty$.

In this note, we shall prove some theorems on the generalized process (1.2), and define the type of the random spectra of (1.2).

The process of type (1.2) is defined as follows:

$$
x(t, \omega)=\sum_{n=1}^{\infty} a_{n}(\omega) e^{i \lambda n(\omega) t}{ }^{1)}
$$

where

i $)^{\prime}\left\{a_{n}(\omega)\right\}(n=1,2, \cdots \cdots)$ is a sequence of mutually independent real random variables with mean values zero, and

$$
\sum_{n=1}^{\infty} E\left\{{a_{n}}^{2}(\omega)\right\}<\infty ;
$$

ii $)^{\prime}\left\{i_{n}(\omega)\right\}(i=1,2, \cdots \cdots)$ is a sequence of mutualy independent real random variables with distribution functions

$$
\left\{F_{n}(x)\right\}(n=1,2, \cdots \cdots) \text { respectively ; }
$$

iii $)^{\prime}$ a system of $\left\{a_{i n}(\omega)\right\}(n=1,2, \cdots \cdots)$ is independent to a system of $\left\{\lambda_{n}(\right.$ (i) $\left.)\right\} \quad(\boldsymbol{n}=1,2, \cdots \cdots)$.

i $\left.)^{\prime}, \mathrm{ii}\right)^{\prime}$ and $\left.\mathrm{iii}\right)^{\prime}$ suffice for the existence of (1.2) with probability 1

1) On this type of a process, the author and G. Marurama have obtained another results which are not yet published. 
and in the sence of mean on the space $\Omega$ for each $t$. And hereafter, we call the process of the type (1.2) a random frequency process $(r . f . p$.$) .$

\$2. Stationality and type of spectral distribution of r. f.p. (1.2).

Theorem 1. The r.f. p. $x(t, \omega)$ is stationary in the weak sence.

\section{Proof.}

Let $R(t, s)$ be the auto-correlation function of $r . f . p . x(t, \omega)$, then

$$
\begin{aligned}
& R(t, s)=E\{x(t, \omega) \overline{x(s, \omega)}\} \\
= & E\left\{\sum_{n=1}^{\infty} a_{n}(\omega) e^{i \lambda_{n}(\omega) t} \sum_{m=1}^{\infty} a_{m}(\omega) e^{-i \lambda_{m_{m}}(\omega) s}\right\} \\
= & E\left\{\sum_{n=1}^{\infty} \sum_{m=1}^{\infty} a_{n}(\omega) a_{m}(\omega) e^{i t \lambda_{\lambda^{2}}(\omega)} e^{-i s \lambda_{m}(\omega)}\right\} .
\end{aligned}
$$

By the independency of iii $)^{\prime}$

$$
\begin{aligned}
& =\sum_{n=1}^{\infty} \sum_{m=1}^{\infty} E\left\{a_{n}(\omega) a_{m}(\omega)\right\} E\left\{e^{i t \lambda_{n}(\omega)-i s \lambda_{m}(\omega)}\right\} \\
& =\sum_{n=1}^{\infty} E\left\{a_{n}{ }^{2}(\omega)\right\} E\left\{e^{i \lambda_{n}(\omega)\{t-s\}}\right\} \\
& =\sum_{n=1}^{\infty} E\left\{a_{n}{ }^{2}(\omega)\right\} \int_{-\infty}^{\infty} e^{i x(t-s)} d F_{\lambda_{n}}(x) \\
& =\sum_{n=1}^{\infty} E\left\{a_{n}{ }^{2}(\omega)\right\} f_{n}(t-s) .
\end{aligned}
$$

Here $\left\{f_{n}\right\}(n=1,2, \cdots \cdots)$ is the characteristic function of distribution function $F_{n}(x)$ respectively.

Q. E. D.

\section{Corollary}

$\left(1^{\circ}\right)$. There can be a r.f.p. $x(t, \omega)$ with a continuous spectral distribution. $\left(2^{2}\right)$. There can be a r.f. p. $x(t, \omega)$ with any given auto-correlation functionof a stationary type.

\section{Proof.}

We can easily see $\left(1^{\circ}\right)$ and $\left(2^{\circ}\right)$ from $(2.2)$, putting $\sum_{n=1}^{\infty} E\left\{a_{n}{ }^{2} \alpha^{(\omega)}\right\}=1$, and $f_{n}(t)=f_{0}(t) \quad(n=1,2, \cdots \cdots)$, where $f_{0}(t)$ is a certain characteristic function which is continuous.

This corollary shows the generalization of Khintchine's method ${ }^{2}$ [1] on constructing the stationary process with a given spectral distribution function.

2) The braçket denotes the number of the biblography. 


\section{Unitary representation and integration of stationary process.}

In this paragraph, we shall give several lemmas on a representation and a definition of integral of a stochastic process for next paragraph after K. KaRUhNen [2] and K. Nagabhushanam [3] .

Let $W$ be a set of real numbers, and let $\sigma(s)$ be a measure defined on the subsets $(s)$ of $W$. Let $Z(s, \omega)$ be a random set function defined on the subsets of $W$ such that, if $s_{1}$ and $s_{2}$ are two disjoint sets,

$$
Z\left(s_{1}, \omega\right)+Z\left(s_{2}, \omega\right)=Z\left(s_{1}+s_{2}, \omega\right) \text {. }
$$

Without loss of generality we may assume

$$
E\{Z(s, \omega)\}=0 .
$$

If for any measurable sets $s_{1}$ and $s_{2}$

$$
E\left\{Z\left(s_{1}, \omega\right) \overline{Z\left(s_{2}, \omega\right)}\right\}=\sigma\left(s_{1}, s_{2}\right)
$$

such a set function $Z(s, \omega)$ is called a "random spectral function". The process $Z(s,(1))$ is also referred to as an orthogonal process. In our later considerations, $W$ consists of the interval $(-\infty, \infty)$ on the real axis. In such a case we shall denote the random set function corresponding to $(-\infty, \lambda)$ by $Z(\lambda,(i))$.

If for all $s$ in $W E\left\{Z(s, \omega)^{2}\right\}$ is bounded, then the process $Z(s, \omega)$ is of bounded norm and $\sigma(W)$ is totally finite. For a process of this type, one can define following Karhunen [2] the integral

$$
\int_{u} f(\lambda) d Z(\lambda, \omega)
$$

as the limit in the mean (1.i.m) of the corresponding Rieman-Stieltjes sums for any complex valued function $f(\lambda)$ of the variable $\lambda$ such that

$$
\int_{w} \mid f(\lambda)^{2} d \lambda
$$

is bounded.

The following theorem of MARUYAMA [4]-CRAmER [5] deals with the representation of a stationary process in the form of an integral of the type just mentioned. That is, supposed that $x(t, \omega)$ is a stationary stochastic process in the weak sense with mean value zero, $x(t, \omega)$ has the representation

$$
x(t, \omega)=\int_{-\infty}^{\infty} e^{i t \lambda} d Z(\lambda, \omega),
$$

where $Z(\lambda,(1))$ is a random spectral function which is defined from $x(t, \omega)$, and the inverse formulue holds: 


$$
Z(\lambda, \omega)=\lim \operatorname{i.m}_{T \rightarrow \infty} \frac{1}{2 \pi} \int_{-T}^{T} \frac{1-e^{-i t \lambda}}{i t} x(t, \omega) d t
$$

§4. The type of random spectrum of the r.f.p. $x(t, \omega)$. Theorem 2 .

$\left(1^{\circ}\right)$ The random spectrum $S(\lambda, \omega)$ of $r . f . p . x(t, \omega)$ has the form

$$
S(\lambda, \omega)=\sum_{n=1}^{\infty} a_{n}(\omega) \Psi_{n}(\lambda, \omega)
$$

where

i) $\left\{a_{n}(\omega)\right\}(n=1,2, \cdots \cdots)$ is a system of the random coefficient of r.f.p. $x(t, \omega)$.

ii) Each $\Psi_{n}(\lambda, \omega)$ of $\left\{\Psi_{n}(\lambda, \omega)\right\}(n=1,2, \cdots, \cdot)$ is a random function which is independent to a system $\left\{a_{n}(\omega)\right\}(n=1,2, \cdots \cdots)$ and whose individual fnnction is a monotone non-decreasing step function which takes values $(0$, and 1$),(-1 / 2$ and $1 / 2)$ or $(-1$ and 0$)$ only.

$\left(2^{\circ}\right)$ Conversely, if the random spectrum $S(\lambda, \omega)$ of a stationary stochastic process $x(t, \omega)$ has the form

$$
S(\lambda, \omega)=\sum_{n=1}^{\infty} a_{n}(\omega) \Psi_{n}(\lambda, \omega),
$$

where

$\mathrm{i})^{\prime}\left\{a_{n}(\omega)\right\}(n=1,2, \cdots \cdots)$ is a system of mutually independent real random variables with mean values zero, and $\sum_{n=1}^{\infty} E\left\{a_{n}{ }^{2}(\omega)\right\}<\infty$.

ii)' same as ii) of $\left(1^{\circ}\right)$,

then $x(t, \omega)$ can be represented as a random frequency process.

Proof.

Ad $\left(1^{\circ}\right)$ : From $(3.7)$ in $\$ 3$, the random spectral $S(\lambda, \omega)$ of $r . f . p$. $x(t, \omega)$ is

$$
\begin{aligned}
& S(\lambda, \omega)=\lim _{T \rightarrow \infty} \frac{1}{2 \pi} \int_{-T}^{T^{\prime}} \frac{1-e^{-i t \lambda}}{i t} x(t, \omega) d t \\
& =\lim . \frac{1}{2 \pi} \int_{-T}^{T} \frac{1-e^{-i t \lambda}}{i t} \sum_{n=1}^{\infty} a_{n}(\omega) e^{i \mu_{\mu_{i}(\omega) t}} d t
\end{aligned}
$$

On the other hand, for arbitrary $N, M>0$, we have

$$
\begin{aligned}
& \left|\underset{\substack{T \rightarrow \infty \\
T^{\prime} \rightarrow \infty}}{\lim } E\left\{\int_{-T}^{T} \frac{1-e^{-i t \lambda}}{i t} \sum_{n=1}^{N} a_{n}(\omega) e^{i \mu_{n}(\omega) t} d t \int_{-T^{\prime}}^{T^{\prime \prime}} \frac{1-e^{-i s \lambda}}{i s} \sum_{n=1}^{M} a_{n}(\omega) e^{i \mu(\omega) s} d s\right\}\right| \\
& =\left|\lim _{\substack{T \rightarrow \infty \\
T \rightarrow \infty \infty}} E\left\{\int_{-T}^{T} \int_{-T^{\prime}}^{T^{\prime \prime}}\left[\frac{1-e^{-i \lambda \lambda}}{i t} \frac{1-e^{i s \lambda}}{-i s} \sum_{n=1}^{N} a_{n}(\omega) e^{i \mu_{n}(\omega) t} \sum_{n=1}^{M} a_{n}(\omega) e^{-i \mu_{n}(\omega) s}\right]\right\} d t d s\right|
\end{aligned}
$$




$$
\begin{aligned}
& =\left|\lim _{\substack{T \rightarrow \infty \\
T, \rightarrow \infty}} \int_{-T}^{T} \int_{-T^{\prime}}^{T^{\prime}} \frac{1-e^{-i t \lambda}}{i t} \cdot \frac{1-e^{i s \lambda}}{-i s} E\left\{\sum_{n=1}^{v} a_{n}(\omega) e^{i \mu_{i}(\omega) t} \sum_{n=1}^{M} a_{n}(\omega) e^{i \mu(\omega) s}\right\} d t d s\right| \\
& \leqq \lim _{\substack{T \rightarrow \infty \\
T^{\prime} \rightarrow \infty}} C \int_{-T^{\prime}}^{T} \int_{-T^{\prime}}^{T^{\prime}}\left|\frac{1-e^{-i t \lambda}}{i t} \frac{1-e^{i s \lambda}}{-i s}\right| d s d t, C \text { : absolute constant. }
\end{aligned}
$$

Therefore, from the calculation just mentioned and the existence of the $\sum_{n=1}^{\infty} a_{n}(\omega) e^{i \mu_{n}(\omega) t}$ with probability $1(\$ 1)$, we obtain

$$
\begin{aligned}
& S(\lambda, \omega)=\sum_{n=1}^{\infty} a_{n}(\omega) \underset{T \rightarrow \infty}{\lim . \operatorname{m}} \frac{1}{2 \pi} \int_{-T}^{T} \frac{1-e^{-i t \lambda}}{i t} e^{i \mu_{n}(\omega) t} d t \\
& =\sum_{n=1}^{\infty} a_{n}(\omega) \Phi_{n}\left\{\lambda, \mu_{n}(\omega), \omega\right\} \\
& \Phi_{n t}\left\{\lambda, \mu_{n}(\omega), \omega\right\}=\underset{T \rightarrow \infty}{\operatorname{iim}} \cdot \frac{1}{2 \pi} \int_{-T}^{T} \frac{1-e^{-i t \lambda}}{i t} e^{i \mu_{26}(\omega) t} d t \\
& =\underset{T \rightarrow \infty}{\lim } \frac{1}{2 \pi} \int_{-T}^{T} \frac{1-e^{t \lambda i}}{i t} \int_{-\infty}^{\infty} e^{i \nu t} d Z_{n}(\nu, \omega) \\
& (n=1,2, \cdots \cdots) \text {. }
\end{aligned}
$$

The last integral is certificated by the fact that each stochastic process $\boldsymbol{e}^{i \mu_{n}(\omega) t}(\boldsymbol{n}=1,2, \cdots \cdots)$ is stationary with respect to a time parameter $t$ and represented as

$$
e^{i \mu}{ }_{n}^{(\omega) t}=\int_{-\infty}^{\infty} e^{i \nu t} d Z_{n}(\nu, \omega), \quad(n=1,2, \cdots \cdots)
$$

by $(3.6)$.

Again, from the inverse formula

$$
\left.\overline{\mathcal{J}}_{n}\left\{\lambda, \mu_{n}(\omega), \omega\right)\right\}=Z_{n}(\lambda, \omega), \quad(\boldsymbol{n}=1,2, \cdots \cdots) .
$$

That is

$$
S(\lambda, \omega)=\sum_{n=1}^{\infty} a_{n}(\omega) Z_{n}(\lambda, \omega) .
$$

Next, we consider $\Psi(\lambda, \mu(\omega), T)$ as follows

$$
\Psi(\lambda, \mu(\omega), T)=\frac{1}{2 \pi} \int_{-T}^{T} \frac{1-e^{-i \lambda t}}{i t} e^{i \mu(\omega) t} d t .
$$

Then

$$
\lim _{T \rightarrow \infty} \Psi(\lambda, \mu(\omega), T)=\lim _{T \rightarrow \infty} \frac{1}{2 \pi} \int_{-T}^{T} \frac{e^{i \mu(\omega) t} \cdot-e^{i\langle\mu(\omega)-\lambda \xi t}}{i t} d t
$$


$=\frac{1}{2 \pi} \int_{-\infty}^{\infty} \frac{\sin \mu(\omega) t-\sin \{\mu(\omega)-\lambda\} t}{t} d t$

1 on the subset $E_{(1)}^{\lambda}$ of $\Omega$ such that $\quad 0<\mu(\omega)<\lambda$ $1 / 2$ on the subset $E_{(2)}{ }^{\lambda}$ of $\Omega$ such that $\left\{\begin{array}{l}\mu(\omega)=\lambda>0 \\ \text { lor } \mu(\omega)=0<\lambda\end{array}\right.$

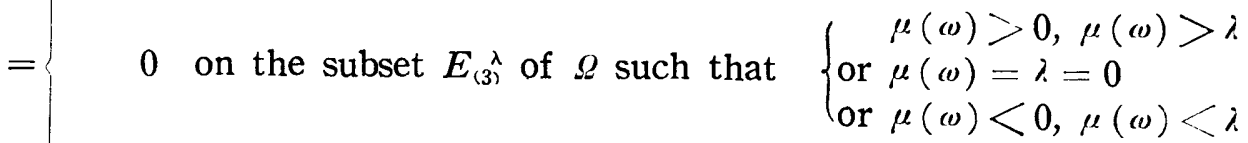

$-1 / 2$ on the subset $E_{(4)}^{\lambda}$ of $\Omega$ such that $\left\{\begin{array}{l}\mu(\omega)=\lambda<0 \\ \text { lor } 0=\mu(\omega)>\lambda\end{array}\right.$

-1 on the subset $E_{(5)}{ }^{\lambda}$ of $\Omega$ such that $\quad 0>\mu(\omega)>\lambda$ $\equiv \Psi(\lambda, \mu(\omega))$.

Here, $\mu(\omega)$ is a real random variable defined on $(\Omega, \mathfrak{F}, P)$, and $\Omega$ is divided as follows

$$
\Omega=E_{(1)}^{\lambda} \oplus E_{(2)}^{\lambda} \oplus E_{(3)}^{\lambda} \oplus E_{(4)}^{\lambda} \oplus E_{(5)}{ }^{\lambda} \oplus N ;
$$

where

$\oplus$ denotes the direct sum, i.e.

$$
E_{(i)}^{\lambda} \cap E_{(j)}^{\lambda}=\Lambda \quad(i \neq j) ; \quad A \text { is empty set, }
$$

and $P(N)=0$.

Now we consider $\Psi(\lambda, \mu(\omega))$ as a random function with a time parameter $\lambda$. Then $\Psi(\lambda, \mu(\omega))$ consists of individual functions such that for any element $\omega_{0} \in \Omega-N$.

a) If $\mu\left(\omega_{0}\right)>0$, individual function

$$
\Psi\left\{\lambda, \mu\left(\omega_{2}\right)\right\}=\left\{\begin{aligned}
1 & \lambda>\mu\left(\omega_{0}\right) \\
1 / 2 & \lambda=\mu\left(\omega_{0}\right) \\
0 & \lambda<\mu\left(\omega_{0}\right)
\end{aligned}\right.
$$

b) if $\mu\left(\omega_{0}\right)=0$, then

c) if $\mu\left(\omega_{0}\right)<0$, then

$$
\Psi\{\lambda, \mu(\omega)\}=\left\{\begin{aligned}
1 / 2 & \lambda>\mu\left(\omega_{0}\right) \\
0 & \lambda=\mu\left(\omega_{0}\right) \\
-1 / 2 & \lambda>\mu\left(\omega_{0}\right)
\end{aligned}\right.
$$

$$
\mathscr{T}\left\{\lambda, \mu\left(\omega_{0}\right)\right\}=\left\{\begin{aligned}
0 & \lambda>\mu\left(\omega_{0}\right) \\
-1 / 2 & \lambda=\mu\left(\omega_{0}\right) \\
-1 & \lambda<\mu\left(\omega_{0}\right)
\end{aligned}\right.
$$

On the other hand,we have 


$$
\begin{gathered}
Z_{n}(\lambda, \omega)=\Phi_{n}\left\{\lambda, \mu_{n}(\omega), \omega\right\} \quad(n=1,2, \cdots \cdots) \\
=\lim _{T \rightarrow \infty} \frac{1}{2 \pi} \int_{-T}^{T} \frac{1-e^{-i t \lambda}}{i t} e^{i \mu_{n}(\omega) t} d t \\
=\lim _{T \rightarrow \infty} \Psi\left(\lambda, \mu_{n}(\omega), T\right) .
\end{gathered}
$$

And, from the existence of $\lim _{T \rightarrow \infty} \Psi\left\{\lambda, \mu_{n}(\omega), T\right\}$ with probability 1 , $Z_{n}(\lambda, \omega)=\Psi\left\{\lambda, \mu_{n}(\omega)\right\}$ with probability $1 . \quad$ Q.E. D.

2) Let $\boldsymbol{x}(\boldsymbol{t}, \omega)$ be a stationary stochastic process with a given random spectre $S_{x}(\lambda, \omega)$ of $(4.2)$. i.e.

$$
\begin{aligned}
& x(t,(1))=\int_{-\infty}^{\infty} e^{i t \lambda} d S_{x}(\lambda, \omega) \\
& S_{x}(\lambda, \omega)=\sum_{n=1}^{\infty} a_{n}(\omega) \Psi_{n}(\lambda, \omega) .
\end{aligned}
$$

Without loss of generality, we can suppose that any individual function $\varphi_{n}(\lambda)$ of $\Psi_{n}(\lambda, \omega)(n=1,2, \cdots \cdots)$ takes value $1 / 2\left\{\varphi_{n}(\lambda+0)+\varphi_{n}(\lambda-0)\right\}$ at the point at which a jump occured.

We induce a sequence of mutually independent random variables $\left\{\mu_{n}(\omega)\right\}$ $(n=1,2, \cdots \cdots)$ from $\left\{\Psi_{n}(\lambda, \omega)\right\}(n=1,2, \cdots \cdots)$ as follows

$$
P\left\{\omega ; \lambda<\mu_{n}(\omega) \leqq \lambda+d \lambda\right\}=P\left\{\omega ; \Psi_{n}(\lambda+d \lambda, \omega)-\Psi_{n}(\lambda, \omega)>0\right\}
$$

In other words, $\left\{\mu_{n}(\omega)\right\}(n=1,2, \cdots \cdots)$ is a sequence of random variables with distribution functions $\left\{F_{n}(x)\right\}(n=1,2, \cdots \cdots)$, respectively, which denotes the measure of individual functions that jump at a point $x$ and points less than $x$.

Then we consider the process $y(t, \omega)$,

$$
y(t, \omega) \equiv \sum_{n=1}^{\infty} a_{n}(\omega) e^{i \mu_{2 s}(\omega) t}
$$

and one can easily see by $(i)^{\prime}$ and (ii) $)^{\prime}$ of (4.2) that

a) the right hand side of (4.13) exists with probability 1 and in the sense of mean on the space $Q$ for each $t$,

b) $y(t$, i $)$ is stationary in the weak sence,

c) the random spectral function $S_{y}(\lambda, \omega)$ of $y(t, \omega)$ is

$$
\begin{aligned}
S_{y}(\lambda, \omega) & =\lim _{T \rightarrow \infty} \frac{1}{2 \pi} \int_{-T}^{T} \frac{1-e^{-i t \lambda}}{i t} \sum_{n=1}^{\infty} a_{n}(\omega) e^{i \mu_{n}(\omega) t} d t \\
& =\sum_{n=1}^{\infty} a_{n}(\omega) \Psi_{n}\{\lambda, \omega\},
\end{aligned}
$$

by the construction of $\left\{\mu_{n}(\omega)\right\}$ and the similar calculation to that of the proof of $\left(1^{n}\right)$. 
Therefore

$$
\begin{array}{rlrl}
y(t, \omega) & =\int_{-\infty}^{\infty} e^{i t \lambda} d S_{y}(\lambda, \omega)=\int_{-\infty}^{\infty} e^{i t \lambda} d\left\{\sum_{n=1}^{\infty} a_{n}(\omega) \Psi_{n}(\lambda, \omega)\right\} \\
& =\int_{-\infty}^{\infty} e^{i t \lambda} d S_{x}(\lambda, \omega)=x(t, \omega) & \text { Q. E. D. }
\end{array}
$$

In conclusion, I wish to express my warmest appriciation of the helpful criticism and encouragement I have reecived from Prof. T. KITAGAwA in the preparation of this paper.

\section{Bibliographies}

1] A. Khintchine. Korrelationstheorie der stationären stochastischen Prozesse, Math. Ann., (1934), p. 608 - p. 610, 112.

$[2] \mathrm{K}$. KaRHUnes. Über lineare Mathoden in der Wahrscheinlichkeitsrechnung, Ann. Ac. Sci. Fennicae, AI 37, Helsinki, (1947). p. 29 - p. 79.

3] K. Nagabrushanam. The primary process of a smoothing relation, Arkiv för Math., (1949) . p. 432 - p. 434.

[4] G. MarUYAMA. The harmonic analysis of stationary stochstic processes, Mem. Fac. Sc., Kyushu Univ., Ser. A, 4, (1949) . p. 71 - p. 73.

[5] H. CRAMÉR. On the theory of stationary random processes, Ann. of Math., 1940. 